\title{
THE LIMIT SETS OF SOME INFINITELY GENERATED SCHOTTKY GROUPS
}

\author{
RICHARD SCHWARTZ
}

\begin{abstract}
Let $P$ be a packing of balls in Euclidean space $E^{n}$ having the property that the radius of every ball of $P$ lies in the interval $[1 / k, k]$. If $G$ is a Schottky group associated to $P$, then the Hausdorff dimension of the topological limit set of $G$ is less than a uniform constant $C(k, n)<n$. In particular, this limit set has zero volume.
\end{abstract}

\section{INTRODUCTION}

Classical Schottky groups are determined by packings of balls in the sphere $\mathbf{S}^{\mathrm{n}}$ and conformal transformations between these balls. A ball packing is a union of round $n$-balls in $\mathbf{S}^{\mathbf{n}}$ such that the interiors of the balls are disjoint. Given a ball packing $P$, a Schottky group associated to $P$ is a group generated by a collection $\left\{C_{b}: b \in P\right\}$ of conformal transformations $C_{b}$ with the following property: To each ball $b \in P$ there is a corresponding ball $b^{\prime} \in P$ so that $C_{b}\left(\mathbf{S}^{\mathbf{n}}-b^{\prime}\right)=\operatorname{int}(b), b^{\prime \prime}=b$, and $C_{b^{\prime}}=C_{b}^{-1}$. A Schottky group is a discrete subgroup of the general Moebius group GM(n). For basic information about Schottky groups, see [B].

The topological limit set $L(G)$ of a Schottky group $G$ is the smallest nonempty $G$-invariant closed subset of $\mathbf{S}^{\mathbf{n}}$. More concretely, as long as $L(G)$ contains more than 2 points $L(G)$ is the set of accumulation points of any orbit. In case $G$ is finitely generated-that is, in case there are only finitely many balls in the packing-much is known about the "size" of $L(G)$. It follows from work of Doyle [D], Phillips-Sarnak [P], and Sullivan [S] that the Hausdorff dimension of $L(G)$ is less than a universal constant $C_{n}<n$. In the infinitely generated case, less is known. One result was obtained by $Z$. X. He, who proved that the limit set of a Schottky group generated by inversions in the disks of a certain type of infinite disk packing has zero area. For a precise statement, see [H].

The result of this paper is essentially a generalization of the those obtained by $\mathrm{He}$, but the methods seem to be different. We say that a packing has $k$ bounded Euclidean geometry if the Euclidean radius of every ball lies in the interval $\left[k^{-1}, k\right]$. Our main result is

Received by the editors November 16, 1990.

1980 Mathematics Subject Classification (1985 Revision). Primary 30F40, 05B40.

Work supported by an NSF Graduate Fellowship. 
Theorem. If $G$ is a Schottky group associated to a packing having $k$-bounded Euclidean geometry, then $\operatorname{dim}(L(G))<C(k, n)<n$.

Here $C(k, n)$ only depends on $k$ and $n$. Taking $n=2$ gives another proof of the fact that a finitely generated classical Schottky group acting on the Riemann sphere has a limit set whose Hausdorff dimension is less than 2. (The finiteness condition guarantees bounded geometry.)

We remark that it is well known that one can produce (infinitely generated) Schottky groups whose limit sets have positive volume, so some sort of restriction on the ball packing underlying the group is necessary.

This paper is organized as follows. In $\S 2$, we give the basic idea of our methods by carrying out the argument in a special case. In $\S 3$, we formalize and generalize the constructions made in $\S 2$. In $\S 4$ we make the analytic estimates needed to prove the theorem.

I would like to thank my teachers Peter Doyle and Bill Thurston for general mathematical help and inspiration, and I would like to thank Dror Bar-Natan and Oded Schramm for helpful discussions about the material in this paper. This paper is my Ph.D thesis, presented at Princeton University in April, 1991.

\section{THE BASIC IDEA}

To illustrate our techniques, we consider the Schottky group $G$ generated by inversions $I_{1}, I_{2}, I_{3}, I_{4}$ in four disks $d_{1}, d_{2}, d_{3}, d_{4}$. We will show here that $L(G)$ has zero area.

Each element $g \in G$ can be written uniquely as

$$
g=C_{i_{1}} \circ \cdots \circ C_{i_{n}}, \quad i_{j} \neq i_{j+1} .
$$

We say that a depth $n$ ball of $G$ is one of the form

$$
C_{i_{1}} \circ \cdots \circ C_{i_{n}}\left(d_{i_{n+1}}\right), \quad i_{j} \neq i_{j+1} .
$$

Let $P_{n}$ denote the set of depth $n$ balls of $G$, and let $\partial P_{n}$ denote the set of spheres bounding these balls. Then the set $\Lambda$ of accumulation points of $\bigcup \partial P_{n}$ is nonempty, closed and $G$ invariant. Therefore $\Lambda \supset L(G)$. In our situation, it suffices to show that $\Lambda(G)$ has zero area.

In our situation, $\partial P_{n}$ consists of finitely many circles, implying that $\Lambda \subseteq$ $\bigcap P_{n} . \quad P_{n+1}$ is obtained from $P_{n}$ by replacing each disk $b \in P_{n}$ by three smaller disks $b_{1}, b_{2}, b_{3} \subset b . \Lambda$ would have area zero if the area of $b_{1} \cup b_{2} \cup b_{3}$ was always a fixed fraction less than the area of $b$. However, as Figure 2.1 shows, this need not be the case.

Looking at Figure 2.1, we see that one of the three smaller disks, say $b_{3}$, might be inconveniently large. Instead of figuring $b_{3}$ into our calculations right away, why not replace $b_{3}$ by its subdivision? This gives us a subdivision of $b$ into the five disks $b_{1}, b_{2}, b_{3,1}, b_{3,2}, b_{3,3}$. This removes one problem, but potentially creates another problem one level down: One of the $b_{3, j}$ might be inconveniently large. So why not iterate this procedure and hope for the best. What remains is a subdivision of $b$ into set $S(b)$ of infinitely many smaller disks, as shown in Figure 2.2. 


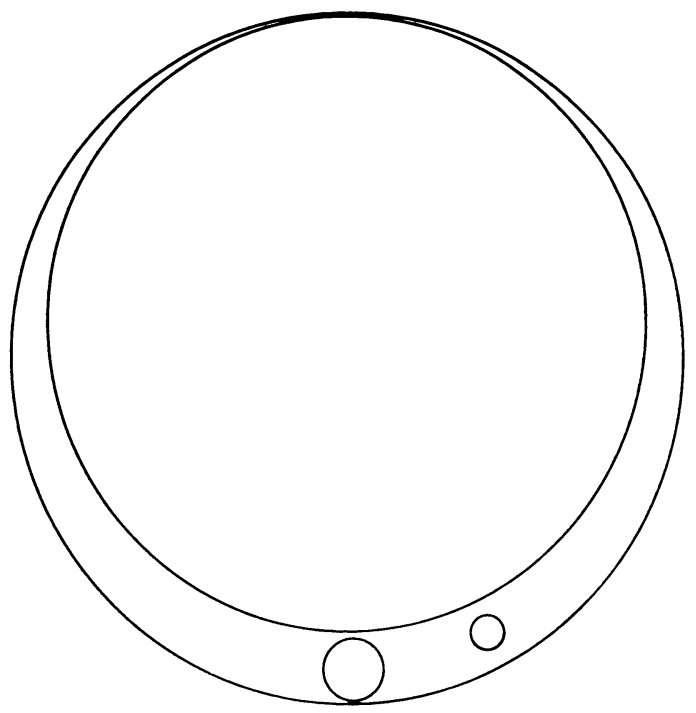

FIGURE 2.1

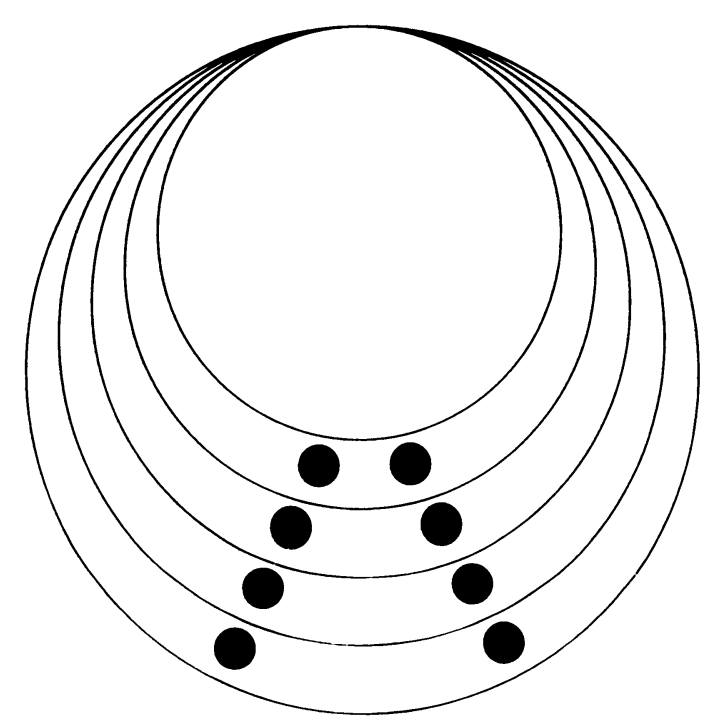

FIGURE 2.2

Define

$$
Q_{1}=P_{1} ; \quad Q_{n+1}=\bigcup_{b \in Q_{n}} S(b) ; \quad Q=\bigcap Q_{n} .
$$

Looking at Figure 2.2, we see that there is an infinite sequence of "crescents", each containing two disks of $S(b)$. The area of the two disks in the crescent is patently a definite fraction less than the area of the crescent. Summing over all crescents tells us that the area of $Q_{n+1}$ is always a fixed fraction less than the area of $Q_{n}$. Hence $Q$ has area zero.

For every $b \in Q_{n}$, let $\Lambda_{b}=\Lambda \cap b$. The set $\Lambda_{b}-S(b)$ is contained in every disk of the nested chain labelled $C$ in Figure 2.2. If this chain shrinks to a point then $\Lambda_{b}-S(b)$ is a single point. If it does not shrink to a point, then it shrinks to a circle, and $\Lambda_{b}-S(b)$ is contained in this circle. In either case 
$\Lambda_{b}-S(b)$ has zero area. By induction

$$
\Lambda-Q_{n+1}=\left(\Lambda-Q_{n}\right) \cup \bigcup_{b \in Q_{n}} \Lambda_{b}-S(b)
$$

has zero area. Therefore, $\Lambda-Q$ has zero area as well.

Putting everything together,

$$
\operatorname{area}(L(G)) \leq \operatorname{area}(\Lambda)=\operatorname{area}(Q)+\operatorname{area}(\Lambda-Q)=0 .
$$

\section{Geometric constructions}

In this section and the next, we fix the dimension $n$ once and for all. Let $\mathbf{D}$ be the unit ball in $\mathbf{E}^{\mathbf{n}}$ and let $I$ be inversion in $\mathbf{D}$.

Subdividing balls. A template is a packing of balls contained in D. Let $\Omega_{k}$ be the set of templates $\omega$ such that $I(\omega) \cup \mathrm{D}$ satisfies the hypotheses of our main theorem. This means that the radius of every ball of $I(\omega) \cup D$ lies in the interval $\left[k^{-1}, k\right]$. We call this the bounded geometry condition. Sometimes it is useful to work with maximal templates. A template is maximal if the addition of any ball produces a packing which is not contained in $\Omega_{k}$. We give $\Omega_{k}$ the Hausdorff topology, considering each template as a point subset of $\mathbf{D}$. This means that two templetes $\omega_{1}$ and $\omega_{2}$ are $\varepsilon$-close if they are contained in the $\varepsilon$-neighborhoods of each other. It is easy to see that $\Omega_{k}$ is compact in this topology. The point is that there are a uniform number of uniformly large balls in any compact subset of $\mathbf{D}-\{0\}$. For convenience, we will assume that the constant $k \geq 1$ has been fixed once and for all. Set $\Omega=\Omega_{k}$.

A subdivision of a ball $e$ is a triple $(\omega, g, e)$ such

(1) $\omega \in \Omega$.

(2) $g \in \mathbf{G M}$.

(3) $g(D)=e$.

Given ball packings $P$ and $P^{\prime}$, we say that $P \prec P^{\prime}$ if $P^{\prime}$ is obtained from $P$ by the subdivision each ball of $P$. An $\Omega$-pattern $P^{*}$ is a sequence of packings $\left\{P_{n}\right\}$, such that $P_{1}$ is a single ball and $P_{n} \prec P_{n+1}$. Define the limit set of $P^{*}$ to be those points $z \in \mathbf{E}^{\mathbf{n}}$ such that every neighborhood of $z$ intersects infinitely many spheres in $\bigcup \partial P_{n}$. We denote this by $\Lambda\left(P^{*}\right)$

Let $G$ be a Schottky group associated to a packing $P$ which has $k$-bounded Euclidean geometry. For each $b \in P$, let $P_{n}(b)$ denote the depth $n$ balls of $G$ which are contained in $b$. Then it is easy to see that the sequence of packings $P^{*}(b)=\left\{P_{n}(b)\right\}$ are $\Omega$-patterns. The templates are all of the form

$$
H_{b} \circ I_{b}(P-b), \quad b \in P .
$$

Here $H_{b}$ is an expansion followed by translation which takes $b$ onto $\mathbf{D}$. Therefore $\Lambda(G)$ is the countable union of the limit sets $\Lambda\left(P^{*}(b)\right)$ together perhaps with the point $\infty=\mathbf{S}^{\mathbf{n}}-\mathbf{E}^{\mathbf{n}}$ (when $G$ is infinitely generated). The upshot is this: in order to bound the Hausdorff dimension of the set $L(G)$, it suffices to bound the Hausdorff dimension of the limit set of an $\Omega$-pattern.

The crescent construction. Suppose $(\omega, g, e)$ is a subdivision. We say that $\beta \in \omega$ is the bad ball if its image under $g$ is the largest. (If there are several, 
then choose arbitrarily.) We define the crescent of the subdivision to be the set $e-g(\omega-\beta)$.

The following lemma is the main compactness tool we use to study the geometry of crescents. Let $\mathbf{G M}(\infty)$ denote the subgroup of $\mathbf{G M}$ which fixes $\infty$ and let $\overline{\mathbf{G M}}=\mathbf{G M} / \mathbf{G M}(\infty)$.

Lemma 1. Suppose $\left\{\omega_{n}, g_{n}, e_{n}\right\}$ is a sequence of maximal subdivisions, having bad ball $\beta_{n}$. Then there is a subsequence (labelled the same way for convenience) so that

(1) $\omega_{n} \rightarrow \omega^{*} \in \Omega$,

(2) $\left[g_{n}\right] \rightarrow\left[g^{*}\right] \in \overline{\mathbf{G M}}$,

(3) $\beta_{n} \rightarrow \beta^{*} \in \omega^{*}$,

(4) $g^{*}\left(\omega^{*}-\beta^{*}\right)$ is contained in a compact subset of $\mathbf{E}^{\mathbf{n}}$.

Proof. (1) follows from the compactness of $\Omega$. (2) follows from the compactness of $\overline{\mathbf{G M}}$, which is homeomorphic to the orthogonal group $\mathbf{O}(\mathbf{n}+1)$.

The only way (3) above could fail was if the size of $\beta_{m}$ was tending to zero. If $\beta_{m}$ is very small, then the bounded geometry condition implies that $\beta_{m}$ is very close to the origin of $\mathbf{D}$. We rescale so that $e_{m}=\mathbf{D}$. We assert that there is a unit diameter ball $B$, whose boundary contains $0 \in \mathbf{D}$, such that $\left|g_{m}^{\prime}(z)\right| \geq\left|g_{m}^{\prime}(0)\right|$ if $z \in B$. If this was the case, then the maximality condition would guarantee that there are some fairly large balls of $\omega_{m}$ having a fairly large intersections with $B$. But the image of such a ball under $g_{m}$ would then be larger than the corresponding image of $\beta_{m}$.

Now we prove the assertion. For simplicity, we prove it for a Moebius transformation $T$ which preserves the unit disk in the complex plane. The general case is similar. In complex notation,

$$
T(z)=\frac{a-z}{1-a z}, \quad a \in(-1,1)
$$

We compute

$$
\left|T^{\prime}(z)\right|=\frac{1-a^{2}}{|1-a z|^{2}} .
$$

Therefore, the level sets of $\left|T^{\prime}\right|$ are concentric circles centered at $1 / a$. In particular, the radius of the level set containing 0 is at least 1 . This proves (3).

Note that (4) above is independent of the coset representative $g^{*}$ of $\left[g^{*}\right]$. Since $g_{n}(\mathbf{D}) \subset \mathbf{E}^{\mathbf{n}}, g^{*}$ cannot take an interior point of $\mathbf{D}$ to $\infty$. If $g^{*}(\mathbf{D}) \subset \mathbf{E}^{\mathbf{n}}$ then there is nothing to prove. We may therefore assume that either $g^{*}(p)=\infty$ for some $p \in \partial \mathbf{D}-\omega^{*}$ or $g^{*}(p)=\infty$ for some $p \in \omega^{*}$. These cases are shown in Figure 3.1. In the first case, $g^{*-1}(\infty) \notin \omega^{*}$. The bounded geometry condition implies that the inverse image of a whole neighborhood of $\infty$ misses $\omega^{*}$ as well, which means all of $\omega^{*}$ is in fact mapped to a compact subset of $\mathbf{E}^{\mathbf{n}}$. 


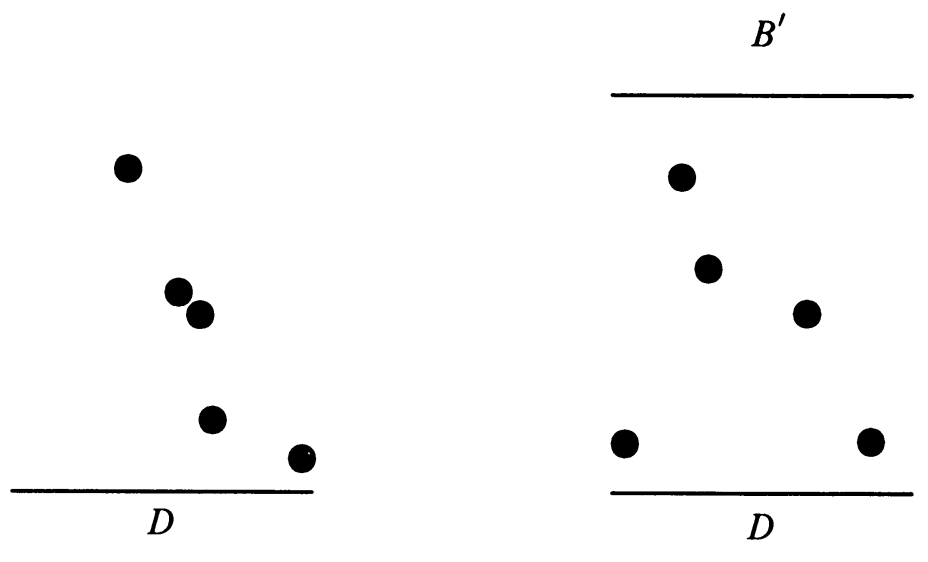

FIGURE 3.1

In the second case, there is a unique ball $\beta^{\prime} \in \omega^{*}$ tangent to $\partial \mathbf{D}$ at $p$, and again the bounded geometry condition implies that there are no other balls of $\omega^{*}$ in a neighborhood of $p$. Hence $g\left(\omega^{*}-\beta^{\prime}\right)$ is contained in a compact subset of $\mathbf{E}^{\mathbf{n}}$. The bad balls $\beta_{n}$ must be getting unboundedly large and hence $\beta_{n} \rightarrow \beta^{\prime}$, implying that $\beta^{\prime}=\beta^{*}$.

We use the same notation as in Lemma 1 . Let $C$ be the crescent of the maximal subdivision $(\omega, g, e)$. Also, the constants $c_{j}$ only depend on the dimension $n$ and the bounded geometry constant $k$.

Corollary 1. For some $c_{1}<1$, volume $(g(\omega-\beta))<c_{1}$ volume $(e)$.

Proof. Suppose we could find a sequence of subdivisions $\left(\omega_{n}, g_{n}, e_{n}\right)$ so that the best constant $c_{n}$ in the inequality above tended to 1 . By Lemma 1 we can take a subsequence and consider the limits $\omega^{*}$ and $g^{*}$. If $g^{*}(D)$ is a finite ball in $\mathbf{E}^{\mathbf{n}}$, then $\left(g^{*}, \omega^{*}, g^{*}\left(\omega^{*}\right)\right)$ is just another subdivision, forcing $\lim \left(c_{n}\right)=\delta<1$. On the other hand, if $g^{*}\left(\omega^{*}\right)$ is not a finite ball, then (4) of Lemma 1 says that $c_{n} \rightarrow 0$.

Corollary 2. If $S \subseteq \omega-\beta$, then

$$
\frac{\operatorname{volume}(S)}{\operatorname{volume}(\omega-\beta)} \in\left[c_{1}, c_{2}\right] \frac{\operatorname{volume}(g(S))}{\operatorname{volume}(g(\omega-\beta))} .
$$

Proof. By integration, the inequality above follows from the assertion that the quantity

$$
\delta(\omega, g, e)=\frac{\sup _{z \in \omega-\beta}\left|g^{\prime}(z)\right|}{\inf _{z \in \omega-\beta}\left|g^{\prime}(z)\right|}
$$

is uniformly bounded. The argument for this is virtually identical to the argument in Corollary 1. There are essentially three main points. First, $\delta$ is independent of scaling. Second, (4) of Lemma 1 guarantees that the distortion of $g^{*}$ on $\omega^{*}-\beta^{*}$ is finite. Third, it is easy to see that $\delta$ varies continuously with subdivisions in the obvious product topology.

For the crescent $C$, define $w(C)$ to be the length of the longest line segment which has endpoints in $\partial(e)$ and $\partial(\beta)$ and which is perpendicular to both. $w(C)$ measures the width of the crescent. 
Corollary 3. volume $g(\omega-\beta)<c_{3} w^{n}(C)$.

Proof. Note that this inequality scales correctly. Suppose that $\left(\omega_{n}, g_{n}, e_{n}\right)$ are a sequence of counterexamples. If we rescale so that $e_{n}$ converges to a finite ball $e$, then we must have $g_{n}\left(\beta_{n}\right)$ converging to $e$ as well. But then, taking limits as in Lemma 1 , we would have to have to have the second case pictured in Figure 3.1. The point is that (rescaling again) the balls $g_{n}\left(\beta_{n}\right)$ and $e_{n}$ are becoming parallel half-planes, which trap a small cylinder containing $g_{n}\left(\omega_{n}, \beta_{n}\right)$. The volume of this cylinder varies like the $n$th power of its width.

Covering the limit set. Let $P=\left\{P_{m}\right\}$ be an $\Omega$-pattern. We say that $b$ is a ball of $P$ if for some $n, b \in P_{n}$. Note that every ball of $P$, except the largest, arises as the subdivision of some other ball of $P$. We say that a bad ball of $P$ is a ball which is the image of the bad ball of some subdivision of another ball. A bad chain is an infinite sequence of balls $\left\{b_{j}\right\}$ such that $b_{j+1}$ is the bad ball in the subdivision of $b_{j}$. Given any ball $b \in P$ we let $\operatorname{bad}(b)$ denote the maximal bad chain contained in $b$. This is exactly the nested sequence of balls shown in Figure 2.2.

We say that a good ball is a ball of $P$ which is not a bad ball. Given any ball $b \in P$, a maximal good ball with respect to $b$ is a good ball contained in $b$ which is not contained in any larger good ball contained in $b$. We let $\operatorname{good}(b)$ denote the set of maximal good balls of $b$. This is exactly the fancy subdivision of $b$ used in Chapter 2.

Define $Q=\bigcap\left\{Q_{n}\right\}$, where

$$
Q_{1}=P_{1}, \quad Q_{n+1}=\bigcup_{d \in Q_{n}} \operatorname{good}(d)
$$

Just as in Chapter 2, we compare $\Lambda=\Lambda(P)$ and $Q$.

Lemma 2. $\operatorname{dim}(\Lambda-Q) \leq n-1$.

Proof. Let $\Lambda_{b}=\Lambda \cap b$. Let $\operatorname{bad}(b)=\left\{b_{1}, b_{2}, \ldots\right\}$, and let $b_{0}=b$. We have the following decomposition:

$$
\Lambda_{b}-\operatorname{good}(b)=\lambda_{\infty} \cup \bigcup_{n=0}^{\infty} \lambda_{n}
$$

where

$$
\lambda_{n}=\left(\Lambda_{b}-\operatorname{good}(b)\right) \cap\left(b_{n}-b_{n+1}\right), \quad \lambda_{\infty}=\left(\Lambda_{b}-\operatorname{good}(b)\right) \cap\left(\bigcap b_{n}\right) .
$$

Consider first $\lambda_{n}$. Let $\left(\omega, g, b_{n}\right)$ be the subdivision of $b_{n} . \lambda_{n}$ consists of those points $z \in b_{n+1}-b_{n}$ such that every neighborhood of $z$ intersects infinitely many balls of $g(\omega)$. If there is such a point $z$, the bounded geometry condition implies that $g^{-1}(z)=0$. Now consider $\lambda_{\infty}$. If $\cap b_{n}$ is a single point, then $\lambda_{\infty}$ is exactly this point. Otherwise $\bigcap b_{n}$ is a ball, and $\lambda_{\infty}$ is contained in the boundary of this ball, which is $n-1$ dimensional.

Now comes the induction step.

$$
\Lambda-Q_{1}=\varnothing, \quad \Lambda-Q_{n+1}=\Lambda-Q_{n} \cup \bigcup_{b \in Q_{n}}\left(\Lambda_{b}-\operatorname{good}(b)\right) .
$$

Therefore $\Lambda-Q_{n}$ is at most $n-1$ dimensional. Taking the union over $n$ gives the result. 


\section{Estimates}

We use the same notation here as in the previous chapter. In this chapter, we prove that $\operatorname{dim}(Q)<C(k, n)<n$. This will complete the proof of our main theorem. In order to use the estimates made in the previous chapter, we make the blanket assumption that every subdivision in our $\Omega$-pattern is a maximal one. There is no problem making this assumption, because adding balls to an $\Omega$-pattern can only increase the size of its limit set.

What needs to be done. From the definition of $Q$, all we need to show is that there is uniformly some $\alpha<1$ and some $p<1$ so that

$$
\sum_{e \in \operatorname{good}(b)} \operatorname{volume}(e)^{p} \leq \alpha \operatorname{volume}^{p}(b) .
$$

Our equation scales correctly, so we can assume that $b$ has unit volume. The good and bad sets below will all refer implicitly to $b$. Let bad $=\left\{b_{1}, b_{2}, \ldots\right\}$. Let $\operatorname{good}_{n}=\operatorname{good} \cap\left(b_{n}-b_{n+1}\right)$, and $\operatorname{good}_{0}=\operatorname{good} \cap\left(b-b_{1}\right)$.

Consider the quantities

$$
d(n, p)=\sum_{e \in \mathrm{good}_{n}} \operatorname{volume}^{p}(e), \quad \bar{d}(n, p)=\operatorname{volume}^{p}\left(\operatorname{good}_{n}\right) .
$$

Also consider the sums

$$
y(p)=\sum_{n=0}^{\infty} d(n, p), \quad \bar{y}(p)=\sum_{n=0}^{\infty} \bar{d}(n, p) .
$$

To prove the main result, it is sufficient to prove the following estimates:

(1) If $c>1$ then for all $p$ sufficiently close to $1, y(p)<c \bar{y}(p)$.

(2) There is some $c^{\prime}<1$ so that for all $p$ sufficiently close to $1, \bar{y}(p)<c^{\prime}$.

The first estimate. To prove (1), it suffices to show $d(n, p)<c \bar{d}(n, p), \forall n$ when $p$ is sufficiently close to 1 . Here is what this means: Given a subdivision $(\omega, g, e)$, having bad ball $\beta$, we just want to see that for $p$ close enough to 1 , we have

$$
\sum_{d \in \omega-\beta} \text { volume }^{p} g(d)<c \text { volume }^{p} g(\omega-\beta) .
$$

In fact, since the inequality above scales correctly, we can assume that $g(\omega-\beta)$ has unit volume. Under these circumstances, we just have to show that

$$
\sum_{d \in \omega-\beta} \text { volume }^{p} g(d)<c
$$

We prove $(*)$ by breaking it into a finite part and an infinite tail end.

Here is the tail end: First of all fix a small $\varepsilon>0$. Let $\omega_{m}$ denote the set of balls $b \in \omega$ so that $I(b)$ is farther than $m$ units from the origin. Because of the bounded geometry condition on $\omega$, there are at most const $m^{n-1}$ balls in $\omega_{m+1}-\omega_{m}$. For $|z|=m$, we have $\left|I^{\prime}(z)\right|=m^{-2}$. Therefore, the volume of $b \in \omega_{m}$ is at most const $m^{-2 n}$ and

$$
\begin{aligned}
\sum_{b \in \omega_{N}} \operatorname{volume}^{p}(b) & =\sum_{N}^{\infty} \sum_{b \in \omega_{k+1}-\omega_{k}} \operatorname{volume}^{p}(b) \\
& \leq \text { const } \sum_{k>N} k^{(n-1)-2 n p} \leq \varepsilon
\end{aligned}
$$


for all sufficiently large $N$ as long as (say) $p \in[3 / 4,1]$. From Corollary 2 of Lemma 1, we have

$$
\frac{\text { volume } g(b)}{\text { volume } g(\omega-\beta)} \leq \text { const } \frac{\text { volume } b}{\text { volume }(\omega-\beta)}
$$

Raising this inequality to the power $p$, we still have

$$
\frac{\text { volume }^{p} g(b)}{\text { volume }^{p} g(\omega-\beta)} \leq \text { const } \frac{\text { volume }^{p} b}{\text { volume }^{p}(\omega-\beta)}
$$

for perhaps a different constant. Therefore,

$$
\begin{aligned}
\sum_{b \in \Omega_{N}} \text { volume }^{p} g(b) & =\sum_{b \in \Omega_{N}} \frac{\text { volume }^{p} g(b)}{\text { volume }^{p} g(\omega-\beta)} \\
& \leq \text { const } \sum_{b \in \Omega_{N}} \frac{\operatorname{volume}^{p} b}{\operatorname{volume}^{p}(\omega-\beta)} \leq \text { const } \varepsilon .
\end{aligned}
$$

The last inequality follows from the fact that the volume of a maximal template is uniformly pinched away from zero.

Here is the finite part: The remaining sum

$$
\sum_{b \in \omega-\omega_{N}-\beta} \operatorname{volume}^{p}(b)
$$

is just a finite sum. By continuity in the exponent $p$, it can be made less than 1 for $p$ sufficiently close to 1 .

Putting both pieces together,

$$
\sum_{b \in \omega_{N}} \text { volume }^{p}(b)+\sum_{b \in \omega-\beta-\omega_{N}} \operatorname{volume}^{p}(b) \leq 1+\text { const } \varepsilon<c
$$

for small enough $\varepsilon$ and $p$ close enough to 1 .

The second estimate. In this section we again assume $p \in[3 / 4,1]$. Recall that we have scaled so that the original ball $b$, the largest ball in our $\Omega$ pattern, has unit volume. Let $\operatorname{bad}(b)=\left\{b_{1}, b_{2}, \ldots\right\}$. Suppose $\left(\omega_{m}, g_{m}, b_{m}\right)$ is the subdivision of the bad ball $b_{m}$. Finally, suppose $C_{m}$ is the crescent of $\left(\omega_{m}, g_{m}, b_{m}\right)$. Our strategy is again to decompose our estimate into a finite part and an infinite tail end.

Here is the tail end:

Lemma 3. Given $\varepsilon>0$, we can choose a uniform $m=m(\varepsilon)$ so that $\bar{y}(p, m) \leq$ $\varepsilon . m$ is independent of the $\Omega$-pattern.

Proof. Consider

$$
\bar{y}(p) \leq c_{1} \sum_{m=0}^{\infty} w^{n p}\left(C_{m}\right) \leq c_{2} \sum_{m=0}^{\infty} w\left(C_{m}\right) \leq c_{3}
$$

Here $c_{j}$ only depends on the dimension $n$ and the bounded geometry constant $k$. The first inequality is just Corollary 3 of Lemma 1 . The second inequality follows from the facts that $w\left(C_{m}\right)<$ const and $n p>1$. A glance at Figure 4.1 explains the third inequality. 

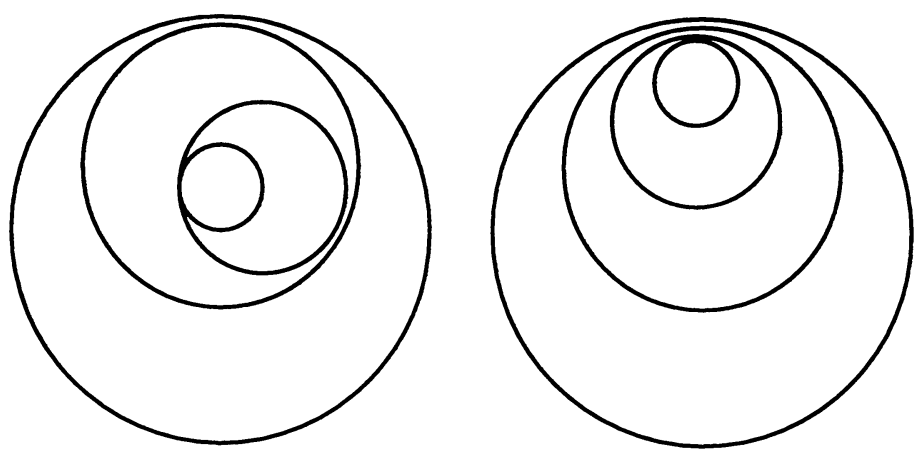

FIGURE 4.1

From $(*)$ it follows that each term of $\bar{y}\left(p, c_{3} / \eta\right)$ is less than $\eta$. It will be useful to set

$$
\eta=\delta^{100 n}
$$

If $\tau_{x}=\operatorname{volume}^{p}\left(\operatorname{good}_{x}\right)$ is a term in the sum $\bar{y}\left(p, c_{3} / \eta\right)$, then there are three possibilities:

(1) $\operatorname{volume}\left(b_{x}\right)<\delta$,

(2) volume $\left(b_{x}\right) \geq \delta$ and $w\left(C_{x}\right)<\delta$,

(3) $w\left(C_{x}\right) \geq \delta$.

We sort the terms of $\bar{y}\left(p, c_{3} / \eta\right)$ into the above three categories.

If there are any terms of type (1) then there is some largest ball $b_{y}$ such that $\tau_{y}$ is a term of type (1). All other terms of type (1) correspond to subdivisions of balls contained in $b_{y}$. Scaling equation $(*)$ tells us that the sum of terms of type (1) is bounded by $c_{3} \delta^{p}$.

For terms of type (2).

$$
\frac{w^{n p}\left(C_{x}\right)}{w\left(C_{x}\right)} \leq \frac{w^{3 / 2}\left(C_{x}\right)}{w\left(C_{x}\right)} \leq \delta^{1 / 2}
$$

Therefore, the sum of type (2) terms is bounded by $c_{3} \delta^{1 / 2}$.

For terms of type (3):

$$
\frac{\tau_{x}}{w^{n p}\left(C_{x}\right)} \leq \frac{\delta^{100 n}}{\delta^{n p}} \leq \delta .
$$

Equation $(*)$ tells us that the sum of terms of type (3) is bounded by $c_{3} \delta$. It is clearly possible to adjust $\delta$ so that the sum of the three terms above is less than $\varepsilon$.

Here comes the finite part of our estimate: Corollary 1 of Lemma 1 says:

$$
\frac{\operatorname{volume}\left(\operatorname{good}_{m}\right)}{\operatorname{volume}\left(b_{m}-b_{m+1}\right)}<\delta_{1}<1 \text {. }
$$

As in Chapter 2, we sum over the crescents: $\bar{y}(1)<\delta_{2}<1$. In particular,

$$
\bar{y}(1)-\bar{y}(1, m)<\delta_{2}<1 \text {. }
$$

Since this last sum is a finite one, we can choose $p$ close enough to 1 so that

$$
\bar{y}(p)-\bar{y}(p, m)<1-2 \varepsilon .
$$


We complete the proof of estimate (2) by combining this last equation with Lemma 3 , which is valid for any $p \in[3 / 4,1]$.

Putting it all together. Let us put everything together. Suppose $G$ is a Schottky group which is associated to a packing having $k$-bounded geometry, and suppose $L(G)$ is the topological limit set of $G$. We can find an $\Omega_{k}$-pattern $P$ such that $\operatorname{dim} L(G) \leq \operatorname{dim} \Lambda(P)$. In the notation above, we write $\Lambda=(\Lambda-Q) \cup Q$.

From the above two estimates it follows that, for some $p<1$, we have

$$
\sum_{b \in Q_{m}} \text { volume }^{p}(b)<\text { const } \alpha^{m} .
$$

Since $\alpha^{m} \rightarrow 0, \operatorname{dim}(Q) \leq n p$. From Lemma $2, \operatorname{dim}(\Lambda-Q) \leq n-1$. Therefore

$$
\operatorname{dim}(\Lambda)=\max (n p, n-1)<c(n, k)<n .
$$

This completes the proof of the main theorem.

\section{REFERENCES}

[B] Alan Beardon, The geometry of discrete groups, Springer-Verlag, 1983.

[D] Peter Doyle, On the bass note of a Schottky group, Acta Math. 160 (1988), 249-284.

[F] Gerald Folland, Real analysis: Modern techniques and their applications, Wiley, 1984.

[H] Zheng-Xu He, An estimate for the hexagonal circle packing, Ph.D. Thesis, 1988.

[P] R. Phillips and P. Sarnak, The Laplacian for domains in hyperbolic space and limit sets of Kleinian groups, Acta Math. 155 (1985), 173-241.

[S] Dennis Sullivan, The density at infinity of a discrete group of hyperbolic motions, I.H.E.S. Publications, 1978.

Department of Mathematics, Princeton University Princeton, New Jersey 08544 Current address: Mathematical Sciences Research Institute, 1000 Centennial Drive, Berkeley, California 94720 